\title{
Nonlinear femtosecond pulse propagation in an all-solid photonic bandgap fiber
}

\author{
Bertrand Kibler, ${ }^{1, *}$ Tadeusz Martynkien, ${ }^{2}$ Marcin Szpulak, ${ }^{2}$ Christophe Finot, ${ }^{1}$ Julien \\ Fatome, ${ }^{1}$ Jan Wojcik, ${ }^{3}$ Waclaw Urbanczyk, ${ }^{2}$ and Stefan Wabnitz ${ }^{1,4}$ \\ ${ }^{1}$ Institut Carnot de Bourgogne, UMR 5209 CNRS-Université de Bourgogne, 21078 Dijon, France \\ ${ }^{2}$ Institute of Physics, Wroclaw University of Technology, 50-370 Wroclaw, Poland \\ ${ }^{3}$ Laboratory of Optical Fiber Technology, Maria Curie-Skłodowska University, 20-031 Lublin, Poland \\ ${ }^{4}$ Dipartimento di Elettronica per l'Automazione, University of Brescia, 25123 Brescia, Italy \\ *Bertrand.Kibler@u-bourgogne.fr
}

\begin{abstract}
Nonlinear femtosecond pulse propagation in an all-solid photonic bandgap fiber is experimentally and numerically investigated. Guiding light in such fiber occurs via two mechanisms: photonic bandgap in the central silica core or total internal reflection in the germanium doped inclusions. By properly combining spectral filtering, dispersion tailoring and pump coupling into the fiber modes, we experimentally demonstrate efficient supercontinuum generation with controllable spectral bandwidth.
\end{abstract}

(C)2009 Optical Society of America

OCIS codes: (190.4370) Nonlinear optics, fibers; (060.2400) Fiber properties.

\section{References and links}

1. F. Luan, A. K. George, T. D. Hedley, G. J. Pearce, D. M. Bird, J. C. Knight, and P. St. J. Russell, "All-solid photonic bandgap fiber," Opt. Lett. 29(20), 2369-2371 (2004).

2. G. Bouwmans, V. Pureur, A. Betourne, Y. Quiquempois, M. Perrin, L. Bigot, and M. Douay, "Progress in solid core photonic bandgap fibers," Opt. Quantum Electron. 39(12-13), 949-961 (2007).

3. Q. Fang, Z. Wang, L. Jin, J. Liu, Y. Yue, Y. Liu, G. Kai, S. Yuan, and X. Dong, "Dispersion design of all-solid photonic bandgap fiber," J. Opt. Soc. Am. B 24(11), 2899-2905 (2007).

4. T. P. White, R. C. McPhedran, C. Martijnde Sterke, N. M. Litchinitser, and B. J. Eggleton, "Resonance and scattering in microstructured optical fibers," Opt. Lett. 27(22), 1977-1979 (2002).

5. N. M. Litchinitser, A. K. Abeeluck, C. Headley, and B. J. Eggleton, "Antiresonant reflecting photonic crystal optical waveguides," Opt. Lett. 27(18), 1592-1594 (2002).

6. G. Ren, P. Shum, L. Zhang, X. Yu, W. Tong, and J. Luo, "Low-loss all-solid photonic bandgap fiber," Opt. Lett. 32(9), 1023-1025 (2007).

7. A. Isomaki, and O. G. Okhotnikov, "Femtosecond soliton mode-locked laser based on ytterbium-doped photonic bandgap fiber," Opt. Express 14(20), 9238-9243 (2006).

8. L. Jin, Z. Wang, Q. Fang, B. Liu, Y. Liu, G. Kai, X. Dong, and B.-O. Guan, "Bragg grating resonances in allsolid bandgap fibers," Opt. Lett. 32(18), 2717-2719 (2007).

9. J. Laegsgaard, "Gap formation and guided modes in photonic bandgap fibres with high-index rods," J. Opt. A, Pure Appl. Opt. 6(8), 798-804 (2004).

10. J. M. Dudley, G. Genty, and S. Coen, "Supercontinuum generation in photonic crystal fiber," Rev. Mod. Phys. 78(4), 1135-1184 (2006)

11. A. Fuerbach, P. Steinvurzel, J. A. Bolger, and B. J. Eggleton, "Nonlinear pulse propagation at zero dispersion wavelength in anti-resonant photonic crystal fibers," Opt. Express 13(8), 2977-2987 (2005).

12. S. Dasgupta, B. P. Pal, and M. R. Shenoy, "Nonlinear spectral broadening in solid-core Bragg fibers," J. Lightwave Technol. 25(9), 2475-2481 (2007).

13. W. J. Tomlinson, R. H. Stolen, and A. M. Johnson, "Optical wave breaking of pulses in nonlinear optical fibers," Opt. Lett. 10(9), 457-459 (1985).

14. C. Finot, B. Kibler, L. Provost, and S. Wabnitz, "Beneficial impact of wave-breaking for coherent continuum formation in normally dispersive nonlinear fibers," J. Opt. Soc. Am. B 25(11), 1938-1948 (2008).

15. B. Kibler, J. M. Dudley, and S. Coen, "Supercontinuum generation in photonic crystal fiber: Influence of the frequency dependent mode area," Appl. Phys. B 81, 337-342 (2005).

16. J. Laegsgaard, "Mode profile dispersion in the generalized nonlinear Schrödinger equation," Opt. Express 15(24), 16110-16123 (2007).

\section{Introduction}

Following its first experimental demonstration [1], the configuration of photonic bandgap fibers consisting in a low-index glass core surrounded by a microstructured cladding 
composed of high-index silica-doped rods has recently been a subject of intense design investigations [2,3]. The inverted index contrast provides a photonic bandgap mechanism, wherein the width and center frequency of the bandgaps is dominated by the resonant properties of individual high-index cylinders [4,5], similarly to anti-resonant reflecting optical waveguides. These all-solid photonic bandgap fibers (all-solid PBGFs) present some of the main advantages of conventional optical fibers, i.e., ease of fabrication and splicing when compared with hollow-core bandgap fibers. Transmission losses as low as $2 \mathrm{~dB} / \mathrm{km}$ have been reported for all-solid PBGFs [6]. Efforts are currently devoted on developing and customizing the specific spectral and dispersive properties which may be required for various applications, such as Bragg gratings and fiber lasers [7,8]. In this Letter we investigate nonlinear femtosecond near-infrared pulse propagation and supercontinuum (SC) generation in an allsolid PBGF. To our knowledge, SC generation has not yet been studied in all-solid PBGFs. We are particularly interested in comparing photonic bandgap (PBG) guiding in the central silica core with total internal reflection (TIR) guiding in the germanium doped inclusions. Indeed, previous works on SC generation in optical fibers are generally based on the modification of the geometric fiber structure properties in order to engineer the dispersion of the guided modes and subsequently favor specific nonlinear effects. Here, the two guiding mechanisms lead to well distinct behaviors in the development of the SC spectral broadening by using the same fiber segment and identical input pulses. Furthermore, we show that the SC generation efficiency is larger at moderate input energies when pumping the large central silica core rather than the small Ge-doped inclusions, owing to a zero group-velocity dispersion (GVD) wavelength within the PBG transmission bandwidth. PBG guiding also allows for easier control of the generated SC bandwidth. Indeed, for the first time to our knowledge, we experimentally demonstrate the impact of PBG guiding on the SC generation process, showing useful insights in generating wavelength-stabilized femtosecond pulses or bandwidth-limited SC generation. The above results are significant for improving the understanding of the nonlinear dynamics of SC spectral broadening in all-solid PBGFs, and for the development of customized SC sources for particular applications.

\section{Fiber properties}

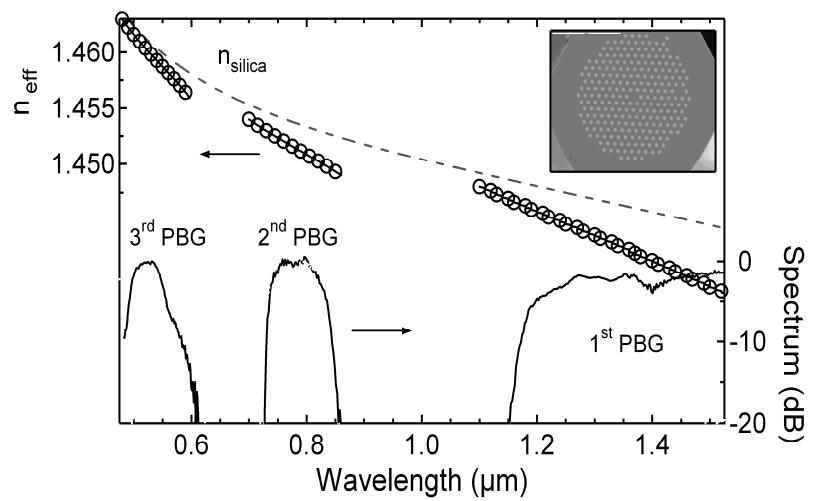

Fig. 1. Numerical calculations of the effective index of the fundamental core mode for the PBGF three bandgaps (circles) compared with the refractive index of silica. The normalized transmission spectrum is obtained by butt-coupling a SC source to $1 \mathrm{~m}$ of the all-solid PBGF. The inset shows the SEM image of the all-solid PBGF. Bright cylinders correspond to Gedoped inclusions.

The all-solid PBGF investigated was manufactured at Maria Curie-Skłodowska University, Poland. It consists of an all-silica core surrounded by 9 rings of Ge-doped inclusions. These inclusions have a parabolic profile with a maximum refractive index difference $\Delta \mathrm{n}=0.03$ (i.e., $\mathrm{GeO}_{2} \sim 20 \%$ ) and a diameter $\mathrm{d}=3.29 \mu \mathrm{m}$, the pitch being $4.82 \mu \mathrm{m}$. The scanning electron microscope (SEM) image of the all-solid PBGF is shown in the inset of Fig. 1. We modeled the all-solid PBGF by means of the finite elements method with perfectly matched 
layer absorbing boundary conditions in order to determine the frequency position of the bandgaps. Our calculations revealed the presence of three bandgaps: one of these bandgaps is centered at $780 \mathrm{~nm}$, which is well-suited for near-infrared pumping. Figure 1 shows the effective index of the fundamental mode calculated for each bandgap, and the transmission spectrum obtained by butt-coupling a supercontinuum source to $1 \mathrm{~m}$ of the all-solid PBGF. These experimental results confirm the presence of three bandgaps, in good agreement with our simulations. Figures $2(\mathrm{a}, \mathrm{b})$ show the wavelength dependence of GVD, loss and nonlinear coefficient of the fundamental PBG core mode in the second bandgap. As expected for allsolid PBGFs, the discrete transmission bands are accompanied by a strong dispersion slope. An important feature of the present all-solid PBGF is that the fundamental core guided mode exhibits a zero dispersion wavelength (ZDW) at $790 \mathrm{~nm}$, near the center of the second bandgap transmission band. At this wavelength, the calculated fiber losses are less than 0.5 $\mathrm{dB} / \mathrm{m}$, whereas the nonlinear coefficient is estimated to be $5.1 \mathrm{~W}^{-1} \mathrm{~km}^{-1}$, as shown in Fig. 2(b). By performing a cut-back measurement, we confirmed that the all-solid PBGF loss is less than $1 \mathrm{~dB} / \mathrm{m}$ near the center of the second bandgap.
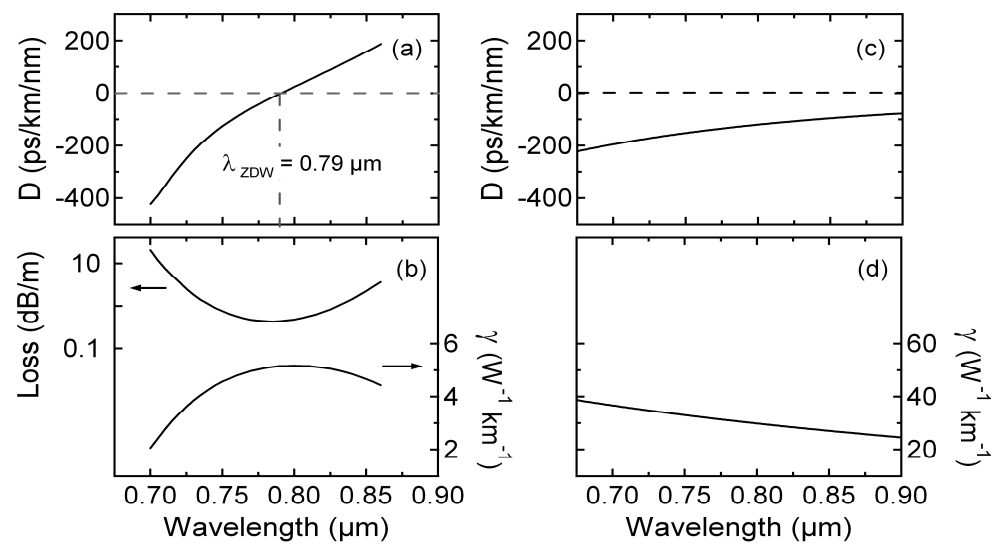

Fig. 2. Numerical calculations of the wavelength-dependent parameters of the fundamental mode for both guiding mechanisms. (a-b) GVD, loss and nonlinear coefficients for PBG guiding in the second bandgap. (c,d) GVD and nonlinear coefficient for Ge-doped inclusion guiding.

\section{Nonlinear pulse propagation}

Nonlinear pulse propagation experiments involved a Ti:Sapphire laser (Mira Coherent) pump source delivering 200 -fs pulses in the wavelength range $750-900 \mathrm{~nm}$ and a $1 \mathrm{~m}$-long segment of the all-solid PBGF. First, we studied nonlinear pulse propagation in the second transmission band of the all-solid PBGF. Figure 3(a) shows the spatial characteristics of light propagating into the silica core, as obtained by imaging the fiber end face with a CCD camera. As it can be seen from the spatial intensity distribution, light is well confined in the central silica core so that it propagates in the fundamental mode. Nevertheless, a small fraction of pump energy is also distributed among several satellite lobes. This typical feature observed in all-solid PBGFs traduces the leaky nature of the PBG core mode and its subsequent mode profile dispersion occurring at the band edges, due to the excitation of inclusions modes [9]. The choice of pump wavelength at $\lambda_{\text {pump }}=785 \mathrm{~nm}$ was motivated by the fact that the largest SC bandwidth is generally obtained by pumping near a fiber ZDW [10]. In our case, experimental and calculated ZDWs are slightly different: from the dynamics of the observed output spectra, we estimated the ZDW to be closer to $785 \mathrm{~nm}$ than $790 \mathrm{~nm}$. Output light spectra were recorded for increasing values of the input peak power, as shown in Fig. 3(c). Inspection of these spectra reveals the typical SC development which is associated with the dynamics of soliton propagation and break-up in the anomalous dispersion regime. Namely, the initial stage of self-phase modulation is followed by pulse break-up and a subsequent soliton self-frequency shift (SSFS) towards longer wavelengths owing to intrapulse Raman 
scattering. Moreover, by pumping close to the ZDW where third-order dispersion is relatively large (with respect to GVD) solitons generated in the anomalous GVD regime shed energy into resonantly coupled radiation or dispersive waves (DW) in the normal GVD regime [10]. Figure 3(c) shows that SC spectral broadening, as induced by both SSFS (long-wavelength edge) and DW generation (short-wavelength edge), grows progressively larger for increasing input power. Eventually this broadening saturates as the PBG transmission band edges are approached. We verified that it was not possible to extend the SC bandwidth beyond the PBG guiding transmission band, irrespective of the pump power or wavelength, as shown in Fig. 4(a) (small discrepancies appear between numerical and experimental results due to the relatively narrower experimental transmission band).
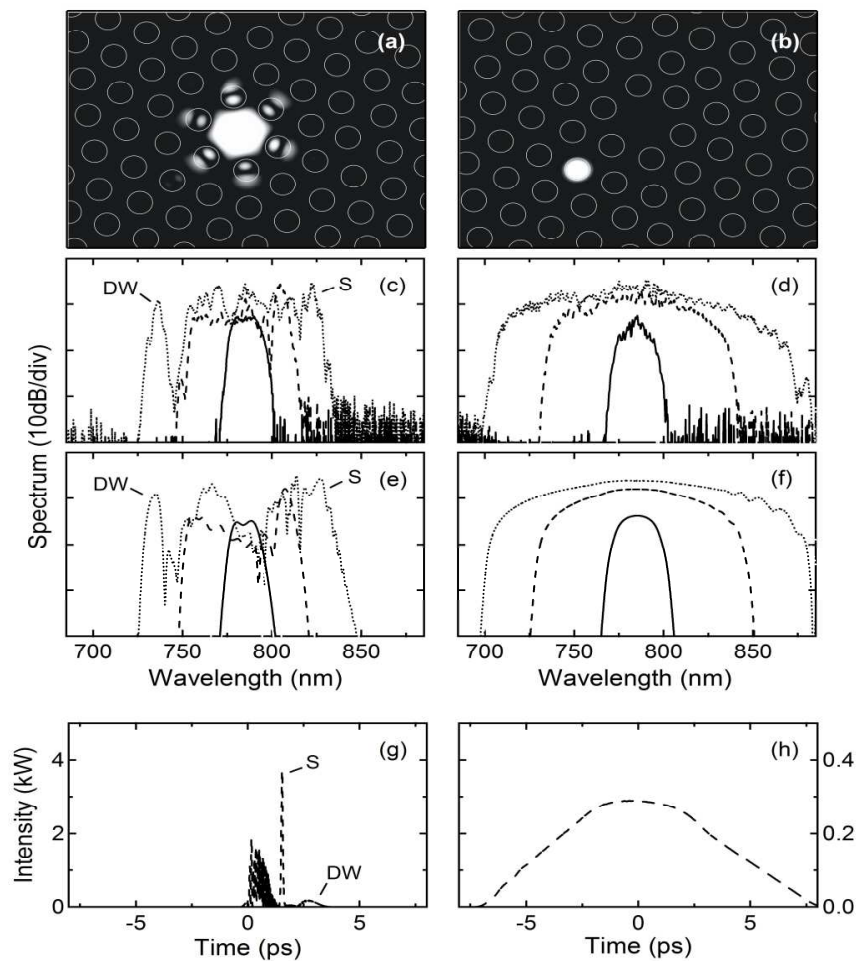

Fig. 3. Observed output mode (a) after PBG guiding into the central silica core, and (b) after TIR guiding into a Ge-doped inclusion. The numerical treatment of another image obtained for wide illumination (of the core and the rods) allowed us to retrieve positions of high index rods, and then superimpose the fiber structure on the mode distribution. Experimental spectra obtained for increasing input peak powers injected into (c) the central silica core $\left(\mathrm{P}_{0}=0.35,2\right.$ and $6.6 \mathrm{~kW})$ and $(\mathrm{d})$ a Ge-doped inclusion $\left(\mathrm{P}_{0}=1,12\right.$ and $\left.28 \mathrm{~kW}\right)$. (e) and (f) Numerical simulations corresponding to (c) and (d), respectively. Output temporal intensity simulated (g) into the central silica core $\left(\mathrm{P}_{0}=6.6 \mathrm{~kW}\right)$ and $(\mathrm{h})$ into a Ge-doped inclusion $\left(\mathrm{P}_{0}=12 \mathrm{~kW}\right)$ associated with similar spectral bandwidths.

By changing the pump wavelength far from the ZDW, we found as expected that the amplitude of the visible peak significantly decreases and eventually it disappears. This fact can be easily explained by considering that for longer wavelengths the spectral overlap between the soliton spectrum and the phase matching wavelength in the normal dispersion regime becomes less efficient [10]. In this case the PBG-induced SC saturation is only observed at the long-wavelength transmission band edge. Indeed, the combined effects of the PBG-induced loss and the strong dispersion slope stabilize the soliton frequency shift to the long-wavelength edge of PBG (a similar effect was observed on DW at the short-wavelength edge). Therefore, the discrete transmission band defines the largest possible SC bandwidth. The interplay among the above described processes (which play a key role in SC generation in 
standard photonic crystal fibers [10]), accompanied by the study and clarification of the impact of PBG guiding on the SC generation process, have not been previously reported in the context of anti-resonant photonic crystal fibers filled with a high index fluid [11]. However, a similar influence of third order dispersion on spectral broadening has been recently reported through numerical studies of nonlinear propagation in solid-core Bragg fibers [12]. Additionally, from a practical point of view (e.g., limited length, instability to temperature and environmental effects, splicing difficulties, etc.), fluid-filled fibers are less suitable for the development of SC sources than the all-solid PBGFs. By customizing their spectral and dispersive properties for particular wavelength-dependent applications, all-solid PBGFs appear to provide a practical alternative to standard photonic crystal fibers for customized SC sources.

To further highlight the impact of PBG guiding on SC generation, we investigated the SC spectral dynamics which are obtained when coupling light into the Ge-doped inclusions. SC spectral broadening may significantly differ in this case owing to the different (i.e, total internal reflection) light guiding mechanism in these doped regions. In particular, one obtains tight mode confinement and large normal GVD in the near-infrared, as reported in Fig. 2(c,d). These parameters of the guided mode in the inclusions were determined by using beam propagation techniques based on a simple step-index profile for the Ge-doped rods. Figure 3(b) shows the imaged near field mode profile when light propagates in a Ge-doped inclusion. As it can be seen, in this case the effective mode area of the excited fundamental mode is significantly reduced with respect to that of the central silica core. As a result, the nonlinear coefficient of the Ge-doped inclusion mode is about 6 times higher than that of the silica core (see Fig. 2(b,d)). We recorded several output spectra for increasing input peak powers in the Ge-doped inclusion, as shown in Fig. 3(d). Just as in the previous case of Fig. 3(c), large spectral broadenings around the pump wavelength are also observed, albeit with significant differences in the spectral shapes. Indeed, the strong normal GVD of the inclusion mode prevents soliton creation: in this case spectral broadening results from the combined effects of self-phase modulation and optical wave-breaking, which is characterized by the generation of significant spectral wings [13,14]. Since light in the inclusion mode is guided by the TIR mechanism and not by the PBG effect, the associated SC spectral broadening is free to extend beyond the PBG transmission band (no SC spectral saturation was observed in this case as shown in Fig. 4(b)). However, in spite of the higher nonlinear coefficient, when coupling the pump into the inclusions the spectral broadening efficiency is reduced (at moderate input pump powers inferior to $12 \mathrm{~kW}$ ) with respect to coupling into the silica core (see also the comparison of SC bandwidths of Fig. 4). The SC generation efficiency is here considered as the capability to generate the largest spectral bandwidth (at -20dB) at comparable input powers and wavelengths. We specify here the need for a customized design of all-solid PBGF to center the transmission band and the zero dispersion wavelength in the vicinity of the pump wavelength in order to obtain a better SC efficiency when compared with nonlinear TIR guiding. These conditions are well satisfied in our work whenever the pump wavelength is set at $785 \mathrm{~nm}$.

In order to confirm our experimental results, we simulated nonlinear pulse propagation in our all-solid PBGF by numerically solving the generalized nonlinear Schrödinger equation (GNLSE) with the split-step Fourier method $[10,15,16]$, including the wavelength-dependent loss, dispersion and nonlinearity. Thanks to the relatively short fiber segment, propagation losses could be neglected for TIR guiding in the doped inclusions. Figures 3(e,f) show the numerical spectra obtained for the same values of input peak power that was used in the experiments. The excellent quantitative agreement between numerical and experimental results confirms the validity of our analysis. The same agreement is also illustrated in the results of Fig. 4, where we display the dependence of the SC bandwidth upon input peak power for two pump wavelengths, i.e., $785 \mathrm{~nm}$ and $815 \mathrm{~nm}$. For PBG guiding in the silica core, as discussed above the SC bandwidth is determined by the interaction of solitons and DWs in the presence of the PBG effect, which shapes the wavelength dependence of GVD and linear losses. Moreover, additional numerical simulations performed without the PBG- 
induced loss contribution confirmed that the strong dispersion slope plays a major role in this spectral saturation [12]. While for TIR guiding in the doped inclusions, we noted that the major spectral broadening occurs over the first ten centimeters of propagation. We have also verified experimentally that the maximum SC bandwidth $(\sim 170 \mathrm{~nm})$ shown in Fig. 4(b) can be generated using only $12 \mathrm{~cm}$ of all-solid PBGF. Such length corresponds to the wave-breaking distance, beyond which the central part the SC spectrum tends to saturate [13]. Such type of pulse spectral broadening in the normal GVD regime has already attracted a great interest in standard optical fibers at telecommunications wavelengths, in particular for spectral slicing applications. Finally, we confirm by the plots of Fig. 3(g,h) that the nonlinear effects which are involved in the presence of each guidance mechanism are vastly different. Indeed, one obtains well-distinct SC temporal properties for similar generated SC bandwidths. PBGguided spectra are generally associated with high-power femtosecond solitons and lowamplitude picosecond DW, while TIR-guided spectra temporally correspond to hugelybroadened and chirped picosecond pulses.
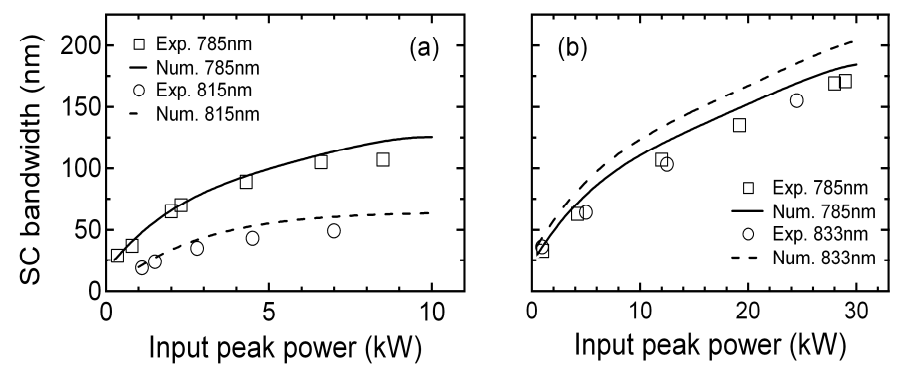

Fig. 4. SC bandwidths (at $-20 \mathrm{~dB}$ ) obtained numerically (lines) and experimentally (markers) for increasing input peak power (a) in the central silica core and (b) in a Ge-doped rod. Two different pump wavelengths were analyzed.

\section{Conclusion}

In conclusion, we have demonstrated the versatile capabilities of a specially designed all-solid PBGF for spectrally tailored SC generation. Indeed, in such fiber two different mechanisms of nonlinear femtosecond pulse guiding are simultaneously present, namely PBG effect in the central silica core and TIR in germanium doped inclusions, respectively. In the first case, efficient and bandwidth-limited SC generation is obtained in a short length of fiber owing to the spectral filtering window containing a ZDW and a strong dispersion slope. These results may provide useful insights for the future development of fiber-based SC sources.

\section{Acknowledgments}

This research was supported by the Agence Nationale de la Recherche (SUPERCODE project, ANR-06-BLAN-0401 ; SOFICARS project, ANR-07-RIB-013-03). We also acknowledge support by the POLONIUM project and the COST 299 Action FIDES. In addition, M. Sz. acknowledges funding from the Foundation for Polish Science - "KOLUMB" Program. 